\title{
Fecundidad y fertilidad de Macrobrachium amazonicum (Héller 1862) (Decápoda, Palaemonidae) del Piedemonte Llanero Colombiano
}

\author{
Fecundity and fertility of Macrobrachium amazonicum (Héller \\ 1862 ) (Decápoda, Palaemonidae) of Colombian \\ Piedemonte Llanero
}

\author{
Elizabeth Aya-Baquero, ${ }^{1 *}$ M.Sc, Yohana Velasco-Santamaría, ${ }^{2}$ Ph.D.
}

\begin{abstract}
${ }^{1}$ Universidad de los Llanos, Facultad de Ciencias Básicas e Ingeniería, Grupo de Investigación Biorinoquia, Villavicencio-Meta, Colombia. ${ }^{2}$ Universidad de los Llanos, Facultad de Ciencias Agropecuarias y Recursos Naturales, Grupo de Investigación sobre Reproducción y Toxicología de Organismos Acuáticos - GRITOX, Villavicencio-Meta, Colombia. Km 12 vía Puerto López, vereda Barcelona, Villavicencio - Meta, Colombia. *Correspondencia: elizabeth.aya@unillanos.edu.co
\end{abstract}

Recibido: Abril de 2013; Aceptado: Agosto de 2013.

\section{RESUMEN}

Objetivo. Determinar la fecundidad y fertilidad de Macrobrachium amazonicum del Piedemonte llanero. Materiales y métodos. Los animales fueron capturados en una granja piscícola del municipio de Restrepo-Meta, Colombia y llevados a la estación piscícola del Instituto de Acuicultura, Universidad de los Llanos. Se utilizaron hembras ovígeras a las cuales se les determinó el peso corporal $(\mathrm{g})$ y longitud total $(\mathrm{mm})$, así como su fecundidad (número de huevos/ hembra) y fertilidad (número de larvas/ hembra). En los huevos se midieron el diámetro y longitud, para calcular su volumen. Resultados. La fecundidad varió entre 120 a 549 huevos con una media de $331 \pm 93$. Con respecto a la fertilidad, las hembras evaluadas tuvieron $287 \pm 19$ larvas, con un intervalo entre 102 a 703 . Tanto para fecundidad como para fertilidad se observó que existe una relación lineal positiva significativa ( $r^{2}$ entre 0.85 y $0.91)$ con respecto al peso corporal y la longitud total de las hembras $(p<0.001)$. El diámetro de los huevos fue de $750.7 \pm 2.9 \mu \mathrm{m}$ (rango: $500-1000 \mu \mathrm{m}$ ) y el largo $974.2 \pm 3.6 \mu \mathrm{m}(525-1200 \mu \mathrm{m})$. El tamaño de los huevos fue de $296.2 \pm 9.7 \mu \mathrm{m}^{3}$. Conclusiones. Los valores de la fecundidad y fertilidad para Macrobrachium amazonicum en el Piedemonte llanero son inferiores a los resultados obtenidos en otros estudios; sin embargo, el tamaño de sus huevos es mayor. Los resultados permiten inferir que la especie se reproduce durante todo el año y que su fácil manejo permitiría su cultivo en cautiverio.

Palabras clave: Camarón de agua dulce, huevos, piscicultura (Fuente: AIMS, FAO). 


\section{ABSTRACT}

Objective. To determine the fecundity and fertility of Macrobrachium amazonicum (freshwater shrimp) of the Piedemonte llanero. Materials and methods. The animals were caught at a fish farm, in the municipality of Restrepo, Meta, Colombia and transported to the fish station of the Institute of Aquaculture of the University of the Llanos. The body weight ( $\mathrm{g})$, and total length ( $\mathrm{mm}$ ) of mature females and the fecundity and fertility (number of eggs and larvae/female, respectively) were determined. The diameter and the length of the eggs were determined to calculate their volume. Results. The fecundity varied from 120 to 549 eggs with an average of $331 \pm 93$. Regarding fertility, the females showed $287 \pm 19$ larvae, with an interval between 102 and 703 . A highly positive lineal regression ( $r^{2}$ between 0.85 and 0.91 ) was observed between fecundity and fertility, and body weight and total length $(p<0.001)$. The egg diameter was $750.7 \pm 2.9 \mu \mathrm{m}$ (range: $500-1000 \mu \mathrm{m}$ ) and egg length of $974.2 \pm 3.6 \mu \mathrm{m}(525-1200 \mu \mathrm{m})$. The egg size was $296.2 \pm 9.7 \mu^{3}$. Conclusions. The determination of fecundity and fertility for Macrobrachium amazonicum in the Piedemonte Llanero was lower to the results obtained in other studies; however, the size of the eggs was higher. The results could infer that the species breeds throughout the year and allows easy handling and farming in captivity.

Key words: Eggs, fish farming, freshwater shrimps (Source: AIMS, FAO).

\section{INTRODUCCIÓN}

Macrobrachium amazonicum (Héller 1862) es un camarón de la familia Palaemonidae con una amplia distribución desde Centro América a Sur América. Short (1) reporta que se han identificado cerca de 210 especies del género Macrobrachium en el mundo, de los cuales 45 están registrados en América Central y del Sur. Dentro de estas, 18 se han identificado en Brasil (2) y en Colombia se reportan un total de 21 especies distribuidas en ríos, lagunas y morichales (3).

M. amazonicum es una especie de importancia comercial en América del Sur debido a su rápido desarrollo y fácil manejo en cautiverio $(4,5)$. Presenta amplia colonización y gran plasticidad adaptativa a los diversos ambientes (6) De igual modo, en Brasil es usado como especie forrajera para peces carnívoros (7). Su tamaño varía de acuerdo al sexo, siendo los machos más grandes con una longitud total de aproximadamente 120 $\mathrm{mm}$; por su parte, las hembras oscilan entre 50 y $100 \mathrm{~mm}$ de longitud total (5). El rostro es alargado con la punta hacia arriba que pasa por el extremo distal del escafocerito. La superficie superior está revestida con 9 a 12 dientes dispuestos irregularmente, de los cuales los primeros siete forman una cresta basal sobre la órbita y la superficie inferior tiene de ocho a diez dientes (2).

El dimorfismo sexual en el género Macrobrachium también puede relacionarse por la presencia del segundo par de quelas, las cuales son más desarrolladas que el resto de apéndices torácicos (1), siendo la quela en los machos más grande que en las hembras. Otra forma de diferenciación es la presencia del poro genital, el cual está ubicado en los machos en el segmento basal del quinto par de pereiópodos, mientras que en las hembras se ubica en el tercer par (2) y estas tienen menos espinas en el segundo par de pereiópodos. Incuban sus huevos en los pleópodos.

Las larvas son planctónicas viven en aguas abiertas o entre macrófitas acuáticas, pastos sumergidos, a profundidades de 0 a $2 \mathrm{~m}$. Las larvas salen a la superficie al caer la noche para aprovechar la mayor cantidad de oxígeno disuelto en el agua y la mayor abundancia de alimentos. Los juveniles son traslucidos y nadan cerca de la superficie del agua, pueden instalarse y caminar sobre sustratos horizontales y verticales. Los adultos son bentónicos y asociados con los sustratos inundados $(7,8)$.

El conocimiento de la fecundidad de una especie es importante para determinar su capacidad reproductiva, el número mínimo de adultos necesarios para mantener el reclutamiento, estimar el tamaño de la población y evaluar su potencial para la acuicultura $(4,5)$. En camarones, la fecundidad se determina como el número de huevos producidos en una época reproductiva y refleja la capacidad de reproducción de cada hembra (5); sin embargo, otros autores la definen como el número de huevos puestos para la eclosión y adheridos a los pleópodos de la hembra (4).

Aunque la fertilidad es el número de larvas producidas por hembra (5); no todos los huevos incubados por una hembra son viables y a veces 
el número de larvas eclosionadas puede ser inferior al número de huevos producidos; por esta razón, es importante evaluar el número de larvas producidas en la eclosión.

Macrobrachium amazonicum tiene valor comercial como cebo para la pesca deportiva, mascota ornamental, alimento vivo para los peces carnívoros ornamentales y para el consumo humano $(9,10)$. Además de su agradable sabor, M. amazonicum es una excelente fuente de proteínas y ácidos grasos esenciales, particularmente EPA y DHA (11).

A pesar de la importancia biológica y económica de $M$. amazonicum no existen suficientes documentos científicos en Colombia que contribuyan al conocimiento de la especie a nivel de su fisiología, reproducción y cultivo, entre otros (7). Para lograr avances importantes en el cultivo del camarón de agua dulce, es necesario estudiar el ciclo vital, la reproducción, nutrición, el crecimiento y el hábitat. A este respecto, en $M$. amazonicum se han realizado estudios orientados a determinar el ciclo reproductivo, maduración gonadal y crecimiento larvario, entre otros (12). Sin embargo, en Colombia solo se conocen estudios sobre la distribución de la especie (3).

Por lo tanto, debido a las limitadas investigaciones en Colombia sobre la biología reproductiva de $M$. amazonicum, el objetivo de este estudio fue estudiar la fecundidad y la fertilidad de la especie y su relación con características biométricas de animales en el Piedemonte Llanero Colombiano.

\section{MATERIALES Y MÉTODOS}

Localización. La colecta de M. amazonicum se realizó directamente en estanques de la piscícola

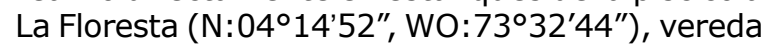
Los Medios, Municipio de Restrepo, Departamento del Meta, Colombia. La piscícola se abastece de agua directamente del río Upin, favoreciendo el ingreso de organismos acuáticos como los camarones. Posterior a la colecta, los animales fueron transportados a la Estación Piscícola del Instituto de Acuicultura de la Universidad de los Llanos, Villavicencio, Meta, Colombia.

Animales experimentales. Las hembras ovígeras de M. amazonicum (Figura $1 \mathrm{~A}$ ) se colectaron de forma manual con la ayuda de una red de $12 \mathrm{~m}$ de largo $\times 1.5 \mathrm{~m}$ de ancho y 0.5 $\mathrm{cm}$ de apertura de ojo de malla. Las capturas se realizaron mensualmente desde Agosto de 2011 hasta Octubre de 2012 ( $n=60$ mensual), excepto en los meses de Abril, Mayo, Junio y Julio, meses en los cuales los estanques fueron desocupados y sometidos a desinfección con cal viva para iniciar nuevamente un ciclo de levante y engorde de peces para el consumo, por tal razón la población de camarones adultos desapareció durante estos meses. En virtud de esta situación, se realizaron varios intentos de captura directamente en el afluente (río Upin), sin embargo, el periodo de lluvias dificultó la consecución de los animales. El traslado de los animales desde el sitio de captura hasta el laboratorio se realizó en bolsas plásticas con agua y oxígeno. Para determinar y establecer taxonómicamente la especie colectada, una muestra de 10 camarones fue llevada al Laboratorio de Crustáceos del Instituto de Ciencias Naturales, Universidad Nacional de Colombia.

Determinación de la fecundidad. Las hembras seleccionadas fueron anestesiadas con $1 \mathrm{ml} / \mathrm{l}$ de 2-fenoxietanol (Sigma-Aldrich), para reducir el estrés durante la manipulación. Un total de 115 hembras ovígeras durante los 10 meses de muestreo se seleccionaron para determinar la fecundidad. La determinación macroscópica del estado de maduración ovárica de $M$. amazonicum fue realizada teniendo en cuenta la coloración, tamaño, posición y apariencia de los ovarios observados a través de la transparencia del caparazón, según los criterios establecidos por $(4,5)$. Adicionalmente, se evaluó el estadio III del huevo caracterizado por forma ovoide, ojos visibles bien desarrollados e intensamente pigmentados (Figura 1B).

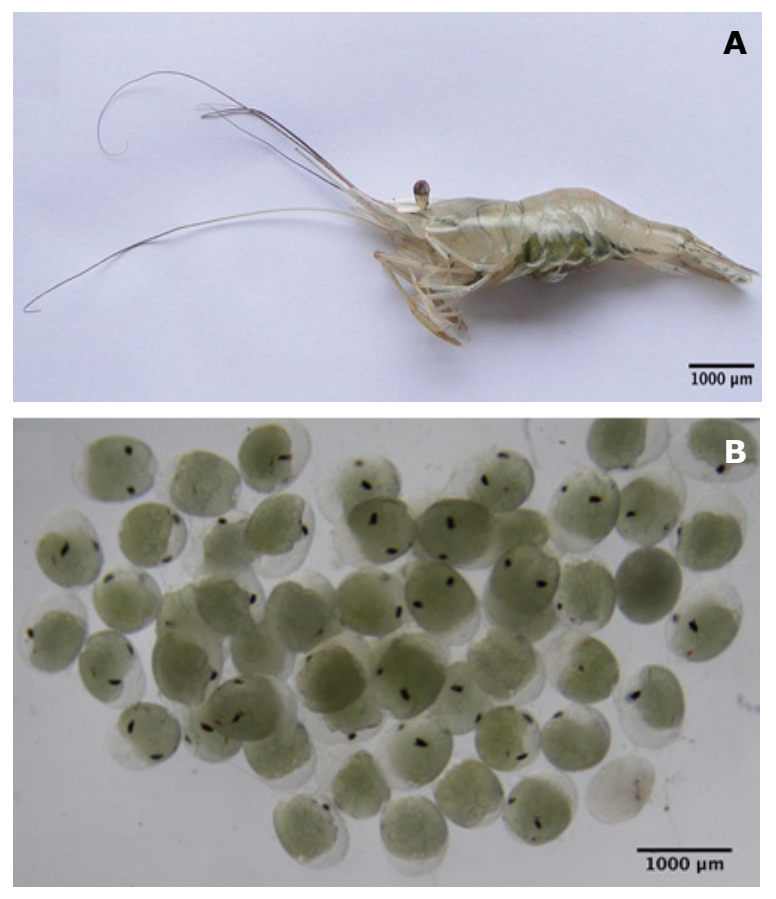

Figura 1. A. Hembra adulta ovígera de camarón de agua dulce M. amazonicum. B. Estadio III del huevo de M. amazonicum. 
Para determinar la longitud total se siguió la metodología propuesta por Parra et al (5) la cual es la sumatoria de la longitud del cefalotórax $(\mathrm{LC})$, longitud del abdomen y del telson $(\mathrm{La}+\mathrm{t})$. Para medir la longitud del cefalotórax (LC), se determinó la distancia entre la extremidad distal del rostro y el punto medio de la parte posterior del caparazón con un calibrador (precisión de $0.05 \mathrm{~mm}$ ). La longitud del abdomen más el telson $(\mathrm{La}+\mathrm{t})$, se determinó como la distancia entre el punto medio superior anterior del primer segmento abdominal y la extremidad distal del telson.

El conteo de los huevos adheridos a los pleópodos de cada hembra, se realizó con la ayuda de pinzas de disección. Luego estos fueron inmersos en solución de Gilson modificada (agua destilada $880 \mathrm{ml}, 100 \mathrm{ml}$ alcohol 60\%, $18 \mathrm{ml}$ ácido acético, $0.15 \mathrm{mg}$ ácido cítrico y $20 \mathrm{mg}$ cloruro de mercurio), y mantenidos durante 15 días para promover la desintegración de las fibras que los adhieren entre sí (Figura 1B). Posteriormente, los huevos fueron contados manualmente en una caja de petri bajo un estereoscopio (Leica EZ4, Alemania. La fecundidad se determinó a partir del conteo total de huevos por hembra. Para determinar la fecundidad promedio, las hembras se agruparon en clases de acuerdo a su peso corporal (entre 1 a $2.4 \mathrm{~g}$, con intervalos de $0.2 \mathrm{~g}$ ) y su longitud total (entre 32 y $53 \mathrm{~mm}$, con intervalos de $3 \mathrm{~mm}$ ). Debido a la diferencia en el peso de las hembras, el número de individuos en cada clase fluctuó entre 2 a 15 y para la longitud entre 2 a 43 individuos.

Para la biometría de los huevos, se midió el largo (L) y el ancho (A) de 973 huevos utilizando un micrómetro ocular calibrado (estereoscopio Leitz), diferenciando los huevos recién fecundados con aquellos que se encontraban en su etapa avanzada, cercana a la eclosión (estadio IV). Por otro lado, para determinar el volumen de los huevos, se utilizó la siguiente formula: $\mathrm{V}=\left(\pi \times L \times A^{2}\right) / 6$.

Determinación de fertilidad. La fertilidad de $M$. amazonicum fue determinada en 78 hembras ovígeras. Una vez en las instalaciones del IALL, las hembras fueron ubicadas en tres acuarios de vidrio con una capacidad de 40 I y con aireación constante. Se utilizó agua de pozo profundo madurada por un periodo mínimo de 8 días. Las condiciones físico-químicas del agua fueron similares a las encontradas en el sitio de muestreo, así: $\mathrm{pH} 6.5 \pm 0.5$; temperatura $27 \pm 1.5^{\circ} \mathrm{C}$, oxígeno disuelto $6 \pm 1.2 \mathrm{mg} / \mathrm{l}$, salinidad 0.2 ppt y conductividad $50 \pm 2 \mu \mathrm{S} / \mathrm{cm}$.

De manera similar a lo realizado para el análisis de fecundidad, las hembras fueron retiradas de los acuarios de 40 I y seleccionadas de acuerdo al color de los huevos (amarillo ocre) y a la presencia de ojos pigmentados, indicativo de estado avanzado de desarrollo embrionario (Figura 1B). Posteriormente, las hembras seleccionadas fueron ubicadas individualmente en acuarios de vidrio de 3 I con el agua previamente madurada y mantenidos con aireación constante. Los animales fueron alimentados una vez al día con ración comercial de $30 \%$ de proteína bruta, en una relación de $10 \%$ de la biomasa. Una vez al día en las horas de la tarde, los acuarios fueron sifoneados para retirar los excesos de alimento y heces con el fin de mantener la calidad del agua; el volumen eliminado fue recuperado adicionando agua madurada.

Diariamente, en horas de la mañana y en la tarde se revisaban los acuarios para determinar el número de larvas eclosionadas en cada acuario. Una vez ocurrida la eclosión, las larvas fueron concentradas en un tamiz de $300 \mu \mathrm{m}$, ubicadas en una caja de Petri y contadas manualmente con ayuda de un estereoscopio y una pipeta Pasteur.

De manera similar a lo realizado para el análisis de fecundidad, en las hembras fue registrada la biometría, agrupando los individuos en categorías de longitud y peso. El peso corporal fue clasificado entre 1 y $2.4 \mathrm{~g}$ con intervalos de $0.2 \mathrm{~g}$ y la longitud total entre 42 y $58 \mathrm{~mm}$, con intervalos de $2 \mathrm{~mm}$.

Otros aspectos reproductivos. Adicional a los parámetros anteriormente expuestos, se realizaron observaciones tanto en las hembras como en la progenie dos veces al día, para determinar las siguientes características: i) Tiempo de incubación: período entre la fertilización de los huevos y la eclosión de los larvas-zoeas. ii) Muda post-eclosión de las hembras: presencia de restos de exoesqueleto en el piso del acuario.

Las larvas-zoeas obtenidas fueron alimentadas con nauplios de Artemia salina recién eclosionada y mantenidas a una dureza de carbonato de calcio y magnesio de aproximadamente $55 \mathrm{mg} / \mathrm{l}$, para estimular las múltiples mudas durante estas primeras etapas.

Análisis estadístico. La estadística descriptiva se realizó para expresar los resultados como media \pm error estándar de la media (SEM). La normalidad se evaluó por medio del test de Kolmogorov-Smirnov. Las clases de peso corporal y longitud total fueron usadas para realizar un análisis de regresión lineal para las variables de fecundidad y fertilidad. Para el tamaño de los huevos se realizó un análisis de frecuencias. Un 
valor de $\mathrm{p}<0.05$ fue utilizado como criterio para considerar diferencias estadísticas significativas. Los datos fueron analizados con el software GraphPad versión 5 para Windows.

\section{RESULTADOS}

El análisis taxonómico de los ejemplares colectados demostró que corresponden a Macrobrachium amazonicum. El material fue incluido en la Colección de Crustáceos, del Instituto de Ciencias Naturales, bajo el numero de catalogo ICN-MHN-CR 2652.

Durante el periodo de aguas altas en la región, entre los meses de Abril a Julio de 2012, no se muestrearon animales, por tanto no hay resultados durante este período. La temperatura promedio fue de $27.5 \pm 0.16^{\circ} \mathrm{C}$, oxígeno disuelto de $5.9 \pm 0.22 \mathrm{mg} / \mathrm{l}$ y $\mathrm{pH}$ de $7.3 \pm 0.11$. Los valores mensuales son mostrados en la figura 2 .

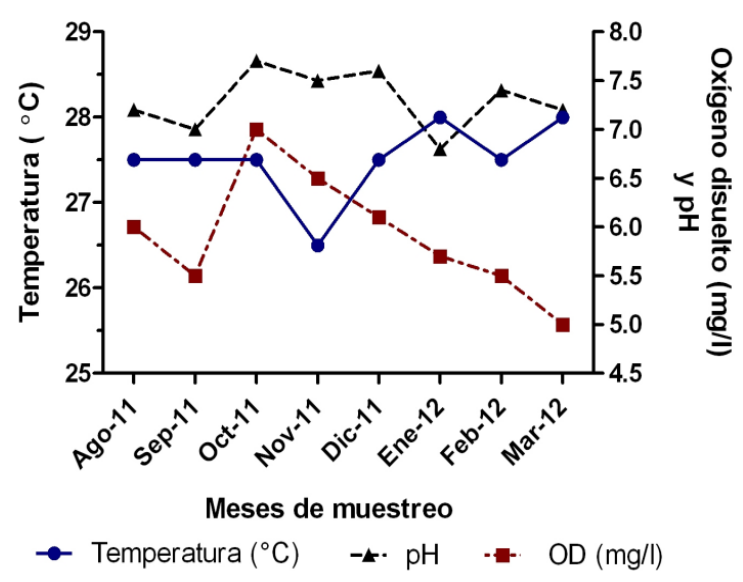

Figura 2. Distribución mensual de temperatura (círculos azules con línea continua), $\mathrm{pH}$ (triángulos negros con líneas discontinuas) y oxígeno disuelto (cuadros rojos con línea discontinua) del agua de la estación piscícola la Floresta, Restrepo, durante los meses de muestreo de M. amazonicum.

Las hembras usadas para evaluar la fecundidad oscilaron entre 32 y $51.8 \mathrm{~mm}$ de longitud total $(43.9 \pm 2.9 \mathrm{~mm})$ y un peso corporal entre $1.0 \mathrm{a}$ $2.3 \mathrm{~g}(1.7 \pm 0.3 \mathrm{~g})$. El rango de fecundidad fue de 120 a 549 huevos con una media de $331 \pm 93$. El análisis de regresión lineal reveló que existe una relación positiva altamente significativa entre la fecundidad y el peso de las hembras $(p=0.0003$, Figura $3 \mathrm{~A}$ ). El coeficiente de determinación indicó que el $91 \%$ de la variación de la fecundidad es explicada por el peso corporal. La ecuación de regresión fue:

Fecundidad $=167.2 *$ peso $(\mathrm{g})+56.34$
De manera similar, se observó una relación positiva altamente significativa entre la fecundidad y la longitud total $(p=0.0028)$, con un coeficiente de determinación 0.86 indicando que el $86 \%$ de la variación de la fecundidad es explicada por la longitud total (Figura 3B). La ecuación ajustada de regresión para fecundidad considerando la longitud fue:

Fecundidad $=10.88 *$ longitud $(\mathrm{mm})-138.4$

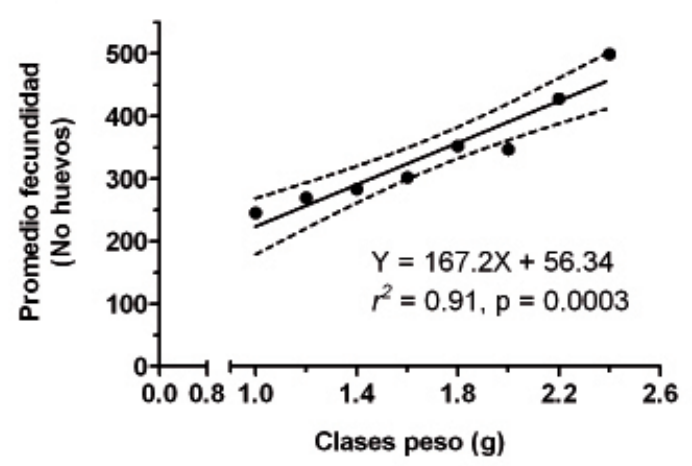

A

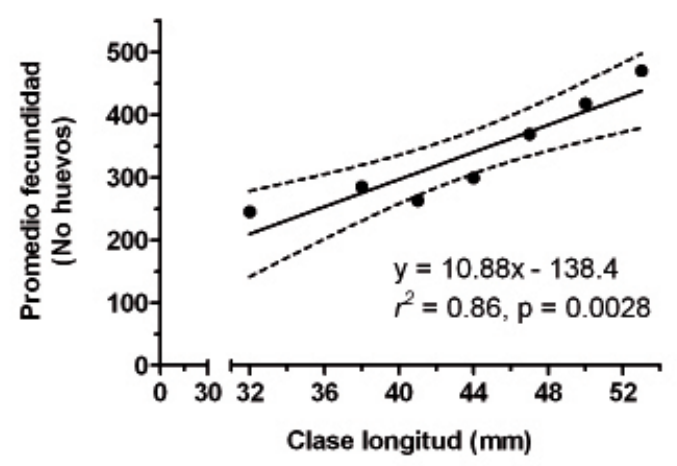

Figura 3. Relación lineal entre la fecundidad (número de huevos) y las clases de A) peso corporal (g) y B) longitud total ( $\mathrm{mm}$ ) de M. amazonicum.

Con respecto a la fertilidad, las hembras usadas oscilaron entre 40 y $58 \mathrm{~mm}$ de longitud total $(48.0 \pm 0.5 \mathrm{~mm})$ y un peso corporal entre 1.0 a $3.1 \mathrm{~g}(1.8 \pm 0.06 \mathrm{~g})$. Las hembras evaluadas tuvieron $287 \pm 19$ larvas, con un intervalo entre 102 a 703. Al igual que la fecundidad, se observó una relación positiva altamente significativa entre la fertilidad y el peso de las hembras (Figura 4A) y la longitud total (Figura 4B).

El coeficiente de determinación indicó que el 86 y el $85 \%$ de la variación de la fertilidad es explicada por el peso corporal o la longitud total, respectivamente $(p<0.05)$.

La ecuación ajustada de regresión lineal para fertilidad fue:

Fertilidad $=170.9 *$ peso $(\mathrm{g})-48.3$

Fertilidad $=26.35 *$ longitud total $(\mathrm{mm})+990.4$ 

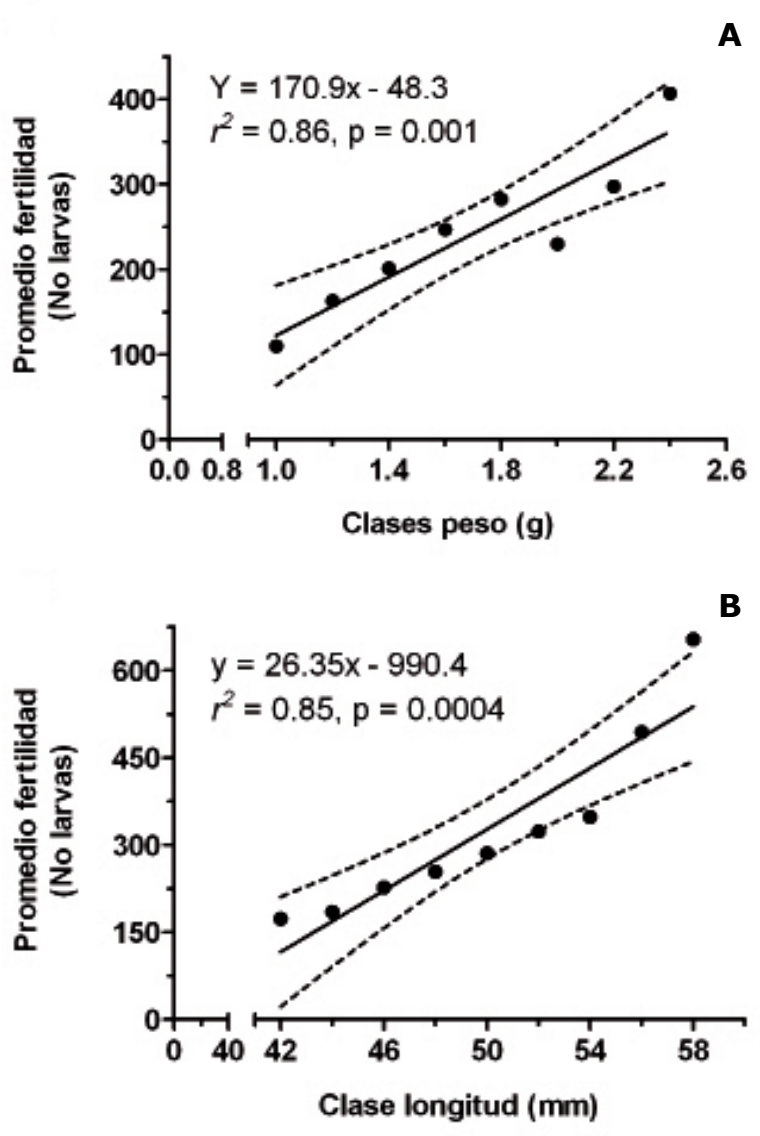

Figura 4. Relación lineal entre la fertilidad (número de larvas) y las clases de A) peso corporal ( $\mathrm{g}$ ) y B) longitud total ( $\mathrm{mm}$ ) de M. amazonicum.

A diferencia de la fecundidad y la fertilidad, el tamaño de los huevos no dependió ni del peso corporal ni la longitud total (regresión lineal, $\mathrm{p}>0.05$ ). El diámetro de los huevos fue de $750.7 \pm 2.9 \mu \mathrm{m}$ (rango: $500-1000 \mu \mathrm{m}$ ) y el largo $974.2 \pm 3.6 \mu \mathrm{m}(525-1200 \mu \mathrm{m})$. El tamaño de los huevos fue de $297.6 \pm 3.2 \mu \mathrm{m}^{3}$ y un rango de 88 a $628 \mu \mathrm{m}^{3}$. La figura 5 ilustra la tendencia del tamaño de los huevos hacia la izquierda, con una mediana de $279.8 \mu \mathrm{m}^{3}$.

El análisis macroscópico de los huevos reveló que a mayor desarrollo embrionario, el tamaño del huevo aumentó.

Adicional a los anteriores resultados, se observaron aspectos importantes en el manejo de M. amazonicum, tales como desove en horas nocturnas, tiempo de incubación de ca. 19 días y muda de las hembras 1 a 2 días después del desove.

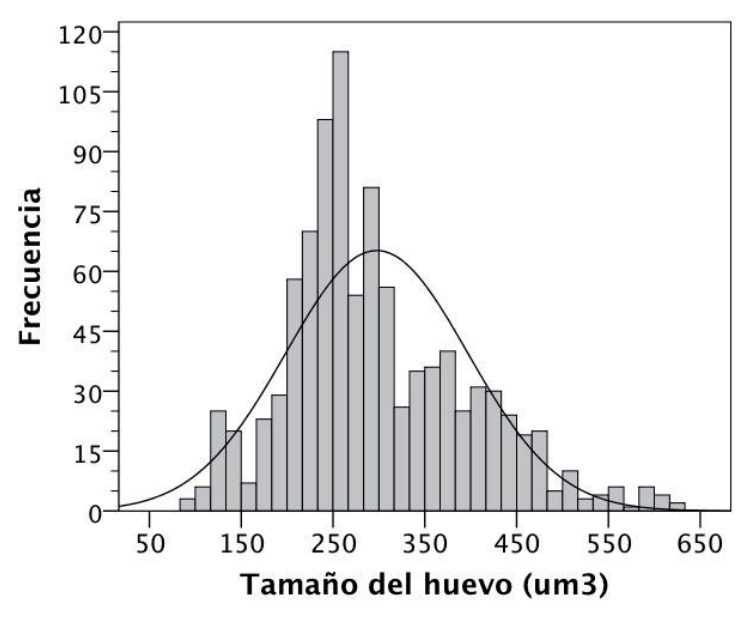

Figura 5. Análisis de frecuencia del tamaño de huevos $\left(\mu \mathrm{m}^{3}\right)$ de M. amazonicum $(n=972)$. Nótese la desviación del tamaño hacia la izquierda.

\section{DISCUSIÓN}

La fecundidad y la fertilidad M. amazonicum se afectó positivamente con las variables de peso corporal y longitud total, sugiriendo que el estado reproductivo aumenta con el tamaño de los camarones.

Da Silva et al (4) y Parra et al (5) afirman que en los crustáceos, la fecundidad puede variar de acuerdo a las condiciones fisiológicas de las hembras, a las estaciones del año, a la latitud, a las condiciones ambientales, la cantidad y calidad del alimento y la temperatura. Estudios realizados han establecido que el rango de fecundidad promedio para M. amazonicum en Suramérica fluctúa entre 102 a 9429 huevos para hembras entre 27 y $110 \mathrm{~mm}$ de longitud total (4, 5-13). La fecundidad absoluta varió entre 40 y 3375 huevos por hembra (14).

La fecundidad es un aspecto reproductivo que depende, entre otros aspectos, del tamaño de las hembras ya que el espacio físico abdominal disponible para portar los huevos es un factor limitante (14). En el presente estudio se observó un rango de fecundidad (120 a 549 huevos) inferior a lo reportado por Parra et al (5), quienes observaron una fecundidad entre 425 y 3274 huevos y por Freire (15) quien reporta una fecundidad de 676 huevos. Esta diferencia puede deberse al mayor tamaño de las hembras ( 0.5 a $4.0 \mathrm{~g}$ y 45 a $75 \mathrm{~mm}$, peso y longitud total, respectivamente) comparado con el tamaño de las hembras del presente estudio. Este hallazgo se soporta con el alto valor del índice de determinación observado entre la fecundidad y el peso y la longitud corporal (Figura 1 y 2 ). En otras 
especies de Macrobrachium (M. heterochirus, $M$. acanthurus, y $M$. carcinus) se ha reportado una relación lineal positiva entre la longitud total y la fecundidad $(16,17)$. En el presente estudio se observó que la fecundidad es directamente proporcional al tamaño de la hembra. Este hallazgo puede deberse a que animales pequeños no tienen el espacio disponible para albergar embriones en desarrollo (15).

La fertilidad de las hembras de M. amazonicum en el presente estudio fue inferior a lo reportado por Parra et al (5), quienes encontraron fertilidades entre 335 a 1435 larvas, equivalente a un promedio de $54 \%$ de fertilidad por hembra. Estos resultados permiten inferir que tanto la fecundidad como la fertilidad son influenciadas positivamente con el peso de las hembras. Esto se ve soportado por los resultados del análisis de regresión lineal en donde el 85 y $86 \%$ de la variación de la fertilidad se explicó por el peso y la longitud total, respectivamente.

El número y el tamaño de los huevos producidos por una determinada especie pueden presentar variaciones debido a las diferencias en la edad y tamaño de los reproductores (7, 15, 17). Similar a los resultados observados en este estudio, Parra et al (5) reporta que a medida que aumenta el largo y el ancho del huevo, avanza el desarrollo embrionario. El tamaño de los huevos de las hembras de $M$. amazonicum fue mayor a lo reportado por Parra et al (5), quienes observaron que los huevos recién fecundados midieron 0.59 y $0.46 \mathrm{~mm}$ de largo y ancho, respectivamente y en etapa de pre-eclosión entre $0.75 \mathrm{~mm}$ y 0.51 $\mathrm{mm}$ de largo y ancho, respectivamente. De igual modo, en un estudio realizado por Ferreira et al (18) se encontró que huevos maduros de $M$. amazonicum tienen un tamaño de $532 \pm 67 \mu \mathrm{m}$, lo cual es relativamente inferior a lo encontrado en el presente estudio. Aunque estos autores no reportaron las tallas de las hembras, muy probablemente los individuos capturados en este estudio fueron más pequeños.
Aunque hay variabilidad en los resultados de fecundidad, fertilidad y biometría de los huevos en poblaciones de M. amazonicum de Colombia, Venezuela y Brasil, estos valores son inferiores a los de otras especies de camarones de interés comercial; sin embargo, según varios autores, el éxito en la colonización y la gran expansión de $M$. amazonicum en las diferentes cuencas es probablemente debido a su gran capacidad de adaptación y la estrategia reproductiva utilizada, dentro de estas su reproducción a través de todo el año, fecundidad relativamente alta y larvas planctotróficas, características adecuadas de esta especie para ocupar los ambientes que tienen cambios drásticos en el régimen hidrológico (17, $19,20)$, lo que permiten que sea una especie de fácil manejo en el laboratorio y por ende de fácil cultivo.

En conclusión los ejemplares capturados mostraron características reproductivas adecuadas a lo largo del año; no obstante, los resultados obtenidos demostraron que tanto la fecundidad como la fertilidad de $M$. amazonicum capturados en el Piedemonte Llanero son inferiores a las reportados en otros estudios, probablemente asociado a su menor tamaño. Por otro lado, el tamaño y el volumen de los huevos obtenidos fueron mayores a lo reportado en esta misma especie bajo condiciones de ambiente natural. Finalmente, a través de este estudio se confirmó las bondades de la especie en términos de reproducción a lo largo del año, docilidad en el manejo, adaptabilidad a las condiciones de cautiverio, lo que la convierte en una especie promisoria para su cultivo comercial.

\section{Agradecimientos}

Este proyecto fue financiado por la Dirección General de Investigaciones de la Universidad de los Llanos (Código FCBI-07-2011). Los autores agradecen al Instituto de Acuicultura de la Universidad de los Llanos por el uso de sus instalaciones.

\section{REFERENCIAS}

1. Short J. A revision of Australian river prawns, Macrobrachium (Crustacea: Decapoda: Palaemonidae). Hydrobiologia 2004; 525(1):1-100.
2. Melo G. (Ed) Manual de identificação dos Crustacea Decápode de água doce do Brasil. Loyola, São Paulo: Universidade de São Paulo; 2003. 
3. Valencia LDM, Rocha de Campos M. Estudio taxonómico de las especies del género Macrobrachium Bate, 1868 (Crustacea: decápoda: Palaemonidae) en Colombia. Acta Biol Colomb 2004; 9(2):122-123.

4. Da Silva RR, Sampaio CMS, Santos JA. Fecundity and fertility of Macrobrachium amazonicum (Crustacea, Palaemonidae). Braz J Biol 2004; 64(3A):489-500.

5. Parra MJC, García SY, Ferrer A, Severeyn H. Aspectos reproductivos del camarón Macrobrachium amazonicum (Heller) en la zona de Nazaret, San Rafael de El Mojón, Lago de Maracaibo, Venezuela. Ciencia 2010; 16(4):402-408.

6. Vergamini FG, Pileggi LG, Mantelatto FL. Genetic variability of the Amazon river prawn Macrobrachium amazonicum (Decapoda, Caridea, Palaemonidae). Contrib Zool 2011; 80(1):67-83.

7. Ramalho MC, Valenti WC. Biology, fisheries and aquaculture of the Amazon River prawn Macrobrachium amazonicum: a review. Nauplius 2009; 17(2):61-79.

8. Marcano N. Aspectos poblacionales del camarón Macrobrachium amazonicum (Héller, 1862) (Decápoda, Palaemonidae) en el río Morocoto, Municipio Benítez, Estado Sucre. Venezuela: Universidad de Oriente, Cumaná, Venezuela; 2006.

9. Bentes B, Martinelli J, Souza L, Cavalcante D, Almeida M, Isaac V. Spatial distribution of the amazon river prawn Macrobrachium Amazonicum (Heller, 1862)(Decapoda, Caridea, Palaemonidae) in two perennial creeks of an estuary on the northern coast of Brazil (Guajará Bay, Belém, Pará). Braz J Biol 2011; 71(4):925-935.

10. Valenti W, Hayd L, Vetorelli M, Martins $M$. Viabilidade econômica da produção de iscas e juvenis de Macrobrachium amazonicum no Pantanal. En: Proceedings of Aquaciência 2006. Bento Gonçalves, RS, Brasil: 2008.

11. Furuya WM, Hayashi C, da Silva AM, Júnior OS, de Souza N, Matsushita M et al. Composição centesimal e perfil de ácidos graxos do camarão-d'água-doce. Rev Bras Zootecn 2006; 35(4):1577-1580.
12. Sampaio CMS, Silva RR, Santos JA, Sales SP. Reproductive cycle of Macrobrachium amazonicum females (Crustacea, Palaemonidae). Braz J Biol 2007; 67(3):551-559.

13. Lobão V, Rojas N, Valenti W. Fecundidade e fertilidade de Macrobrachium amazonicum (Heller, 1862)(Crustacea, Decapoda) em laboratório. Bol Inst Pesca 1986; 13(2):15-20.

14. Lucena-Frédou F, Filho JSR, Silva MCN, Azevedo EF. Population Dynamics of the River Prawn, Macrobrachium Amazonicum (Heller, 1862) (Decapoda, Palaemonidae) on Combu Island (Amazon Estuary). Crustaceana 2010; 83(3):277-290.

15. Freire JL, Marques CB, Silva BB. Estrutura populacional e biologia reprodutiva do camarão-da-amazônia Macrobrachium amazonicum (Heller, 1862)(Decapoda: Palaemonidae) em um estuário da regiao nordeste do Pará, Brasil. Braz J Aquat Sci Technol 2012; 16(2):65-76.

16. Nazari E, Simões-Costa M, Müller YMR, Ammar D, Dias M. Comparisons of fecundity, egg size, and egg mass volume of the freshwater prawns Macrobrachium potiuna and Macrobrachium olfersi (Decapoda, Palaemonidae). J Crust Biol 2003; 23(4):862-868.

17. Lara LR, Wehrtmann IS. Reproductive biology of the freshwater shrimp Macrobrachium carcinus (L.) (Decapoda: Palaemonidae) from Costa Rica, Central America. J Crust Biol 2009; 29(3):343-349.

18. Ferreira MAP, Resende BM, Lima MYS, Santos SSD, Rocha RM. The stage of ovarian development affects organ expression of vitellogenin as well as the morphometry and ultrastructure of germ cells in the freshwater prawn Macrobrachium amazonicum (Heller, 1862). Theriogenology 2012; 78(5):981-990.

19. Silva K, Cintra I, Muniz A. Aspectos bioecologicos de Macrobrachium amazonicum (Heller, 1862) a jusante do reservatório da hidrelétrica de Tucuruí, Pará. Bol Tec Cient Cepnor 2005; 5(1):55-71.

20. Montoya JV. Freshwater shrimps of the genus Macrobrachium associated with roots of Eichhornia crassipes (water hyacinth) in the Orinoco Delta (Venezuela). Caribb J Sci 2003; 39(1):155-159. 